\title{
石墨的纳米结构组装
}

\author{
传秀云 \\ (北京大学 地球与空间科学学院, 北京 100871)
}

摘 要: 本文在分析石墨微观结构和性能基础上, 综合分析了石墨加工改性方法, 提出了石墨纳米结构组装的概念, 介绍了几种石墨纳米结构组装的方法。通过结构组装, 引入纳米功能粒子, 制造活性功能空间, 合成新型石墨功能 材料; 通过制备石墨层间化合物、碳石墨合金等方法引入纳米功能粒子组装碳石墨材料; 通过打开石墨层片, 制备 二维层状材料制备纳米石墨烯片, 可以采用氧化活化等制造孔隙结构增加活性空间; 通过调节石墨晶体排布方向 减少石墨材料的性能异向性, 提高性能均匀性; 通过石墨结构纳米组装设计, 设计新型石墨功能材料。纳米尺度的 石墨加工和改性有可能推动石墨矿物资源的有效利用, 开发新型石墨储能材料和石墨烯片材料。

关 键 词: 石墨; 石墨烯; 二维层状材料; 纳米组装; 综述

中图分类号: TD 98 文献标识码: A

\section{Microstructure Design of Graphite in Nanoscale}

\author{
CHUAN Xiu-Yun
}

(School of Earth and Space Science, Peking University, Beijing 100871, China)

\begin{abstract}
According to microstructural features of graphite, this paper reviewed the processing and dressing methods of graphite and proposed the nanostructured and functionalized engineering concept of graphite principally. The microstructure and properties of graphite could be modified and designed by using several approaches, such as introduction of nanoparticles by both synthesis of graphite intercalation compounds and preparation of graphite alloy. Active functional space for function particles could be induced by pore and porous structure formed through oxidation and activation. It is possible to synthesize graphene like flakes through exfoliation graphite and stripping layered structured graphite, to prepare the layer by layer functional materials. Due to the layered structure, graphite has strong anisotropic properties, which can be decreased by modification of flake direction and then becomes the isotropic graphitic functional materials. It suggests that microstructure design and nanostructured engineering in nanoscale can improve the properties of graphite, and obtain cheaper functional materials from natural graphite by characterization and nanostructure engineering, for wide potential applications, such as energy storage materials and graphene like materials.
\end{abstract}

Key words: graphite; graphene; layer by layer materials; nanostructure; review

天然石墨是重要的战略资源, 中国的石墨储量 和产量都居世界首位 ${ }^{[1-4]}$ 。天然石墨大多只是应用在 相对简单的初加工领域，据统计，大部分天然石墨
只是作为原料，用作粉末冶金增碳剂、高温碳质耐 火材料、工业金刚石、机械制造润滑材料、印刷墨 粉、铅笔制作等等 ${ }^{[4]}$ 。

收稿日期: 2017-01-05; 收到修改稿日期：2017-03-30

基金项目: 国家自然科学基金(51774016, 51274015, 40972027)

National Natural Science Foundation of China (51774016, 51274015, 40972027)

作者简介: 传秀云(1965-), 女, 教授. E-mail: xychuan@pku.edu.cn 
石墨具有优异的导电和导热性能, 具有良好的 化学和高温稳定性, 润滑和涂敷性能优良, 是重要 的非金属矿物资源。本文在分析石墨微观结构、性 能的基础上，综合分析石墨加工改性方法，提出石 墨的纳米组装的概念 ${ }^{[5-6]}$, 并提出多种石墨的纳米 结构组装方法。通过纳米结构组装, 可以制备成新 型石墨功能材料和结构材料, 有可能成为新型储能 材料 ${ }^{[7]}$, 在新兴的新能源汽车、风力发电、环境治理 等行业具有广阔的发展前景和巨大的应用潜力。

\section{1 石墨中碳原子的化学键结构特征}

在原子分子水平上, 石墨中碳原子被杂化, 形 成 $\mathrm{sp}^{2}$ 杂化轨道, 在 $\mathrm{XY}$ 方向上, 碳原子通过共价键 相连形成六方环, 在平面上成层分布, 形成碳原子 层。层面内碳原子之间通过共价键结合, 电子活性 低, 但是层面间只有很弱的分子键存在, 电子活动 性高。这种特殊的结构特征使石墨内部包含丰富的 载流子, 表现出优异的传导性能, 使石墨能够被用 做电极材料、润滑材料、传热材料等 ${ }^{[4,6]}$ 。

\section{2 石墨的纳米结构组装}

可以采用多种方法对石墨进行纳米结构组装: 通过增加功能空间、增加功能粒子 ${ }^{[4]}$, 制备新型石墨 材料, 开发性能良好的石墨制品; 通过制备石墨层 间化合物的方法, 引入纳米功能粒子组装石墨材料; 通过制备石墨合金方法组装石墨材料; 通过引入缺 陷、孔隙结构增加储能空间组装石墨材料; 通过调 节石墨晶体排布方向减少石墨材料的性能异向性, 提高性能均匀性等。

\section{1 石墨层间化合物引入纳米功能粒子组装 石墨新材料}

石墨具有很好的层状结构, 层面内碳原子以 $\mathrm{sp}^{2}$ 杂化轨道电子形成的共价键形成牢固的六角网 状平面, 碳原子间具有极强的键合能 (345 kJ/mol); 而在层间碳原子, 则以微弱的范德华力相结合(键 能 $16.7 \mathrm{~kJ} / \mathrm{mol}$ )。正因为石墨中层面与层间键合力的 巨大差异及微弱的层间结合力, 导致多种原子、分 子、粒子团可以顺利突破层间键合力, 插入层间, 形 成石墨层间化合物(GICs-Graphite Intercalation Compounds $)^{[8-19]}$ 。这些插入物在石墨层内规律排布, 可 以形成规则的阶结构和畴结构等(图 1(a) $)^{[13-19]}$ 。石 墨层间化合物的单层厚度(Identity period)与阶数有

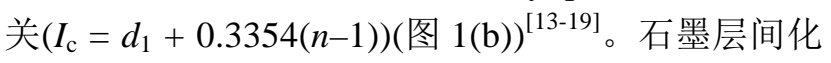
合物可以形成规则的 $1,2,3, \cdots 10$ 阶结构, 形成
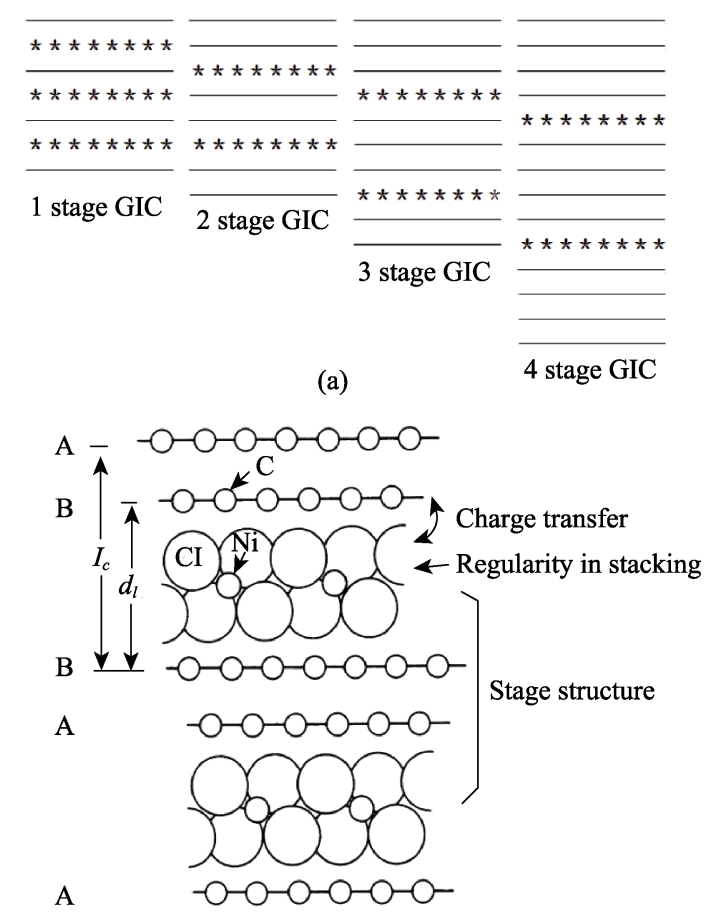

(b)

图 1 石墨层间化合物(GICs)的结构示意图 ${ }^{[15,20]}$

Fig. 1 Sketch of structure and graphite intercalation compound ${ }^{[15,20]}$ (a) Stage structure, - graphite layer; ** intercalate; (b) Layered structure

的石墨层间化合物可以是受主(acceptor)或施主型 (donor)的离子型(Ionic)的插层剂, 也可以是共价型 (Covalent)的插层剂 $(\mathrm{F}, \mathrm{O}+\mathrm{OH})$ 。在石墨层间化合物 中, 插层剂可以双插层(binary)、三插层(ternary)或多 插层。在石墨层间, 插层剂还可以形成局部短程有 序的畴结构。

目前已有 200 多种原子、分子、粒子团能够顺 利突破层间键合力插入层间, 形成多种石墨层间化 合物 ${ }^{[8-19]}$ 。通过石墨层间化合物可以引入纳米功能 粒子, 在石墨微观结构里, 实现纳米功能粒子组装, 创造和提高石墨储能功能, 组装成新的材料, 石墨 层间化合物不但保留了石墨原有的性能, 而且附加 了原有石墨和插层物质均不具备的新性能。插层物 的多少, 在石墨层间的排布规律, 特别是其阶结构、 畴结构等对于石墨层间化合物的性能有决定性作用。

氢的插入有可能使石墨成为储氢材料; 锂离子 在石墨层间的插入和脱插可以实现充放电, 使得石 墨成为性能良好的二次电池材料 ${ }^{[7]}$ 。石墨不仅可以 作为二次锂离子电池负极材料, 而且可以作为一次 电池的正极电池材料, 例如作为锂氟电池正极材 料、高能碱性电池正极导电材料, 以及燃料电池中 双极板材料、核能、太阳能(硅的制备)结构材料等。 锂资源紧缺、价格高, 可以采用资源更加丰富和廉 价的钠离子, 通过合成钠的石墨层间化合物, 制备 
钠离子电池。通过钠离子在石墨层间的插入和脱插 实现充放电, 从而存储能源。氯化铅插层形成的石 墨层间化合物是性能优异的打印墨粉; 澳插层形成 的石墨层间化合物是性能优异的红外屏蔽材料等; 氯化铁等插层形成的石墨层间化合物对毫米波有良好 的衰减性能, 有可能成为毫米波遮蔽干扰屏障材 料 ${ }^{[20-23]}$ 。采用石墨层间化合物可以在石墨碳原子层 间引入纳米功能粒子组装石墨材料, 实现石墨的纳 米组装, 获得优异性能的新材料。

\section{2 碳石墨合金方法引入纳米功能粒子组装} 碳石墨新材料

通过合金方法制备类似于合金的材料, 例如碳 石墨合金方法可以改变碳石墨材料的性能。因为碳、 嗍、氮三种元素在元素周期表中位置靠近, 碳原子 半径与嗍原子、氮原子也相近, 嗍、氮也可能替代 碳石墨材料结构中的碳原子, 形成结构稳定的原子 置换型固溶体, 但是却可以改变石墨原来的性能。

当嗍原子替代碳原子时, 可以形成硼碳合金材 料(图 2), 随着嗍碳比例不同, 调整反应条件, 在一 定温度压力下, 还可以形成 $\mathrm{B}_{50} \mathrm{C}_{2} 、 \mathrm{~B}_{8} \mathrm{C} 、 \mathrm{~B}_{13} \mathrm{C}_{2} 、 \mathrm{~B}_{4} \mathrm{C} 、$ $\mathrm{BC}_{3}$ 等不同组成的嗍碳合金。引入氮原子后, 可以 形成 B-C-N 三元体系 ${ }^{[19,24-25]}$, 获得更多的硼碳氮合

\section{金材料(图 3)。}

通过引入碳、硼、氮等物质形成固溶体, 可以 对石墨进行纳米组装, 改变石墨的性能, 制备新材 料。石墨是导电体, 当嗍原子替代碳原子时, 形成的 嗍碳氮合金却变成了绝缘体或者半导体。嗍碳氮合 金还可以形成不同特征的半导体: $n$ 型半导体、 $p$ 型半导体。

石墨是兼具金属和半金属特性的具有金属光泽 的材料, 当嗍原子替代碳原子时, 形成的硼碳合金 $\left(\mathrm{C}_{x} \mathrm{~B}\right)$ 的金属性更强; 而氮替代形成的硼碳氮 h-BN,

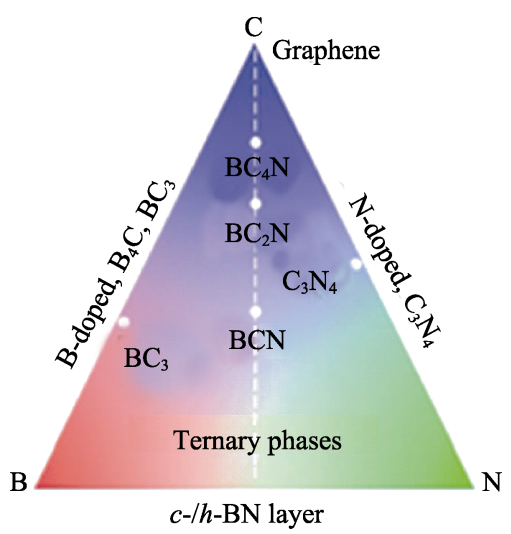

图 2 碳一嗍-氮合金相图 ${ }^{[21]}$

Fig. 2 Diagram segregation of C-B-N phase ${ }^{[21]}$

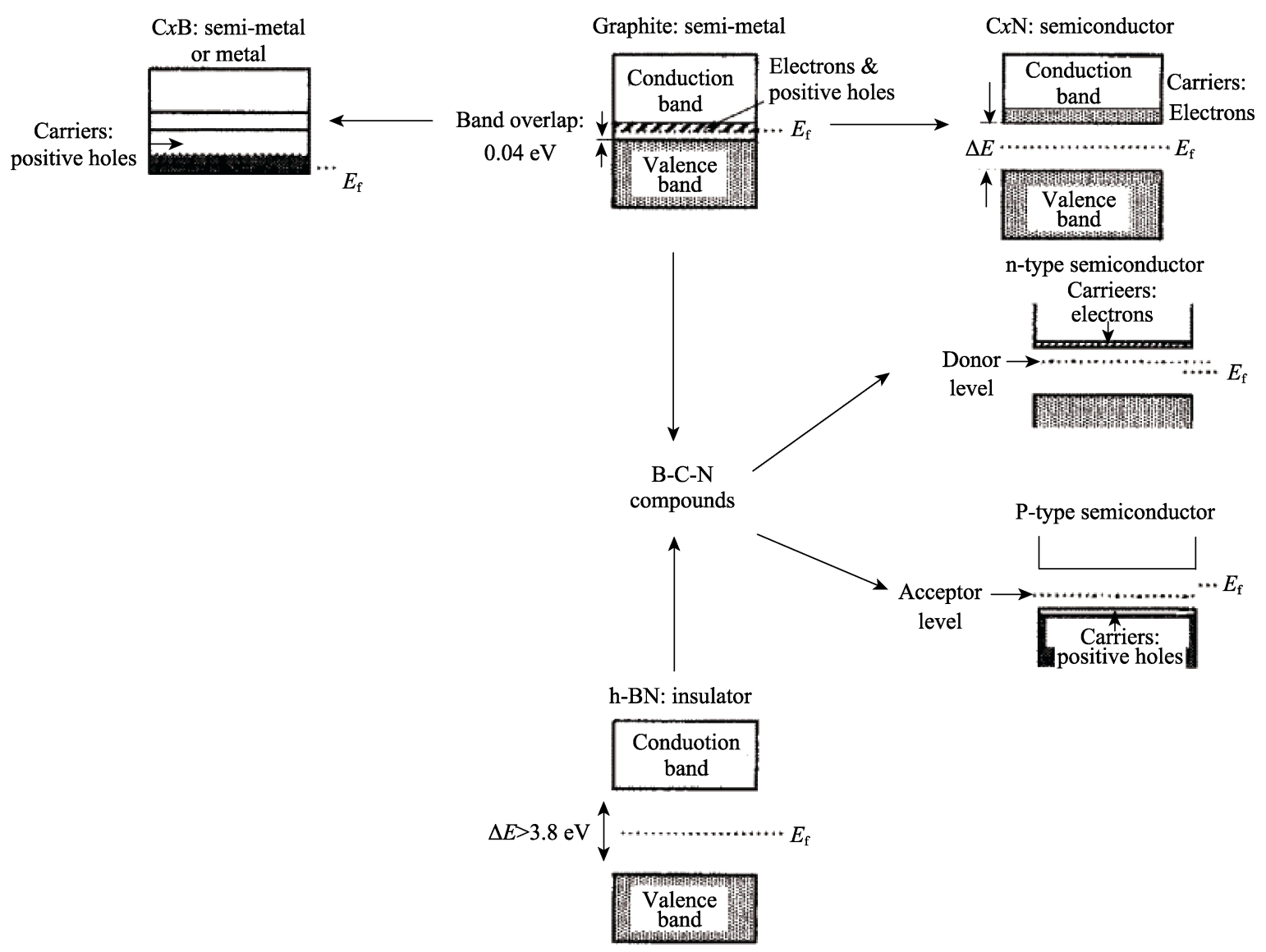

图 3 碳一嗍-氮体系材料的性能变化示意图

Fig. 3 Sketch change of properties of C-B-N materials 
完全不具金属性，成为绝缘体。通过原子置换形式， 用硼一氮等替代石墨中的碳原子, 形成结构稳定的 置换型固溶体。采用这种嗍碳合金方法制备石墨合 金, 可以引入纳米功能粒子组装石墨材料。通过硼 的替换, 已经能够制备出二层、三层的嗍碳氮二维 烯片材料等新型功能材料 ${ }^{[25]}$ 。

\section{3 通过打开石墨层片制备纳米石墨烯片}

理论上, 理想石墨烯是二维晶体, 基本结构就 是标准的碳原子组成的六方网(图 4(a))。根据亮场透 射式电子显微镜下观测到的形貌绘制了处于自由状 态的悬空石墨烯的原子结构示意图(图 4(b)), 单层 石墨烯并非完美的二维平面, 而是在约 10 25 nm 范 围内表面褶皱与水平面局部存在夹角, 褶皱高度可达 $1 \mathrm{~nm}^{[26-30]}$ 。一个独立的碳原子层是石墨烯的理想状态, 石墨烯与三维石墨在结构上的最大差异是其厚度。

理论上, 石墨可以看成是由石墨烯堆叠而成, 石墨烯与石墨的层数界限也成为判断是否是石墨烯 的依据。当碳原子层的层数少于 10 层时, 其电子结 构与普通三维石墨有很大差异 ${ }^{[29]}$, 因此, 碳材料学 界一般将 10 层以下碳原子层组成的材料(Graphene 和 Few-layer graphenes)统称为石墨烯材料(Graphenes), 一般称为单层石墨烯、双层石墨烯和多层石 墨烯 ${ }^{[30]}$ 。

通常状态下，石墨具有鳞片状的片状结构，只 是石墨的鳞片大小厚度有别。理论上将石墨的鳞片 打开, 将本身堆积在一起的石墨碳原子层打开, 就 可以形成单层或多层的石墨烯, 少于 10 层时也被称 为石墨烯。通常很难做到均匀厚度的大片石墨烯, 通常也将获得的纳米尺度薄层石墨称为“纳米石墨 烯片, $[30]$ 。

在强氧化性酸的环境下，石墨易形成石墨层间 化合物。利用石墨这一特性, 将天然石墨置于发烟 硝酸中, 并加入硝基甲烷, 配制成液体炸药, 使用 塑料容器盛装后放入爆轰反应釜中引爆, 收集爆轰 产物, 即得到薄层石墨烯片, 平均厚度达到 $14 \mathrm{~nm}$, 属于多层石墨烯片材料 ${ }^{[31]}$ 。
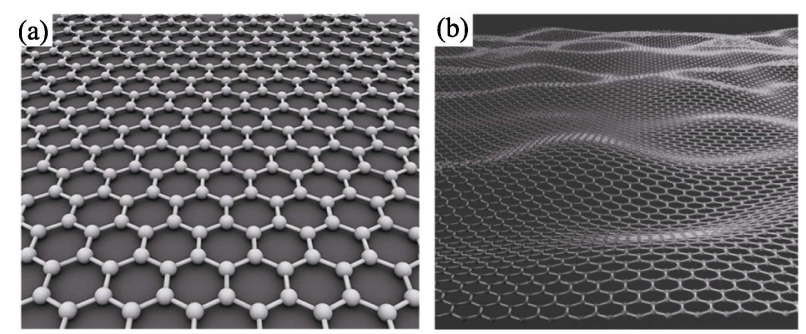

图 4 石墨烯的结构示意图 ${ }^{[14,16]}$

Fig. 4 Sketch of graphene structure ${ }^{[14,16]}$

(a) Ideal structure of single 2D graphene; (b) Waved graphene surface
通过剥离石墨鳞片制备二维层状材料, 可以获 得纳米石墨烯片 ${ }^{[29-30]}$, 单一碳原子层片内很强的共 价键使石墨烯片具有很高的机械强度, 是潜在的力 学结构件材料。这些石墨烯片叶还具有优异的电化 学性能、润滑性能、比表面积大, 是潜在的超高电 容器的材料, 具有良好的应用前景。

石墨烯独特的结构和优良的性能使其在电化学

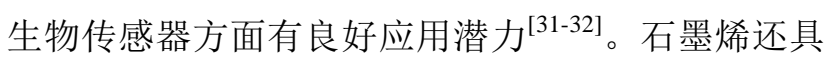
有低细胞毒性、溶解能力强、光致发光稳定等优点, 在酶生物传感器中表现出灵敏度高、选择性好以及 稳定性好等优异性能。石墨烯及其复合物有可能构 建传感系统和生物成像, 在酶传感器、免疫传感器、 DNA 传感器等酶电化学生物传感器内发挥作用。但 是, 石墨烯与酶的作用机制、石墨烯与传感性能的 关系、酶在石墨烯上固载有效性等问题仍有待深入 研究。由于量子限域效应和边界效应, 石墨烯材料 的衍生物一石墨烯量子点有光致发光性能, 在生物 成像、生物传感器等方面有应用潜力 ${ }^{[32]}$, 但是其产 率低、难以精确控制尺寸。新的石墨烯量子点制备 方法、表面修饰方法对于石墨烯及其复合物生物传 感器的发展有重要意义。

\section{4 通过制造孔隙结构增加活性空间}

在石墨结构里制造缺陷也可以对石墨进行结构 组装。引入缺陷最有效的方法就是制造碳原子的空 位, 可以采用氧化活化等方法制造孔隙结构增加活 性空间, 氧化石墨就是一种有效方法。在多孔碳材 料中增加孔隙, 提高比表面积, 能够引入功能空间, 使得锂离子储存量提高, 提高双电层发生空间, 从 而增大了双电层电容器 (Electrical Double-Layer Capacitors-EDLC)的能量存储和转换 ${ }^{[33]}$ 。在石墨中 设法引入孔隙, 增加其比表面、同样也可能增大储 能空间。通过锂离子石墨层间化合物制备的锂离子 电池, 与孔隙效应制备的超级电容器的有机结合, 有可能得到更高功率和容量的储能器件, 大幅度延 长采用清洁能源的新能源电动车工作时间。

通过石墨插层化合物方法, 制备石墨残余石墨 层间化合物, 进而制备成膨胀石墨(图 5(a) ) ${ }^{[13-14]}$ 。采 用硫酸石墨插层混合物高温热处理的方法, 已经能 够大批量制备膨胀石墨。膨胀石墨具有丰富的孔隙 结构, 能够吸收一系列污染物, 治理油类污染 ${ }^{[34-37]}$ 。 膨胀石墨也能被压制成石墨纸, 用作各种耐腐蚀的 密封垫、手机散热片等。采用微波膨胀法可以获得 膨胀效果更好的膨胀石墨(图 5(b) $)^{[29-30]}$, 为更高性能 的膨胀石墨, 甚至石墨烯片的制备提供了更有效的 方法。 

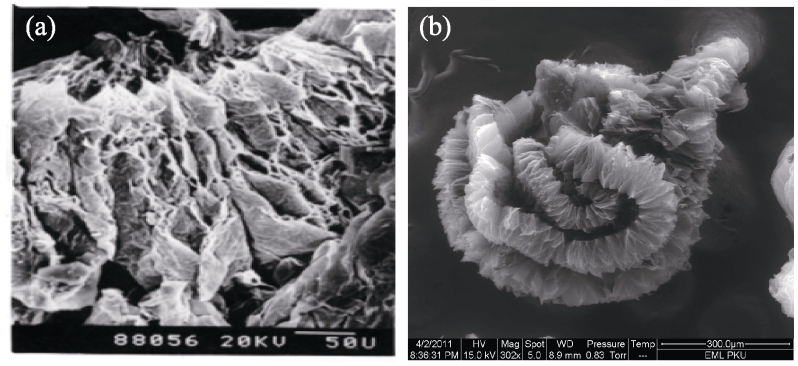

图 5 石墨层间被打开后形成的膨胀石墨的孔隙结构的 SEM 照片 ${ }^{[11,29]}$

Fig. 5 SEM images of pore structure of expanded graphite by exfoliation $^{[11,29]}$

(a) Expanded graphite by heat treatment; (b) Expanded graphite by microwaved treatment

通过在石墨中引入孔隙, 制造孔隙结构增加储 能空间。膨胀石墨中有大量孔隙, 可以在此基础上 制备一系列膨胀石墨复合材料, 例如金属膨胀石墨 复合材料。将膨胀石墨加入到水泥中, 能够制备膨 胀石墨水泥复合材料，这种材料是高弹性、高韧性 建筑材料, 可以提高桥梁的减震性能 ${ }^{[38-39]}$ 。

\section{5 通过调节石墨晶体排布方向减少石墨制 品的性能异向性}

石墨具有层状结构，三维方向上的化学键存在 很大差异，导致单个石墨晶体的性能也具有异向性， 包括力学性能、电学性能、热学性能等。石墨的异 向性有很大的应用价值, 石墨层片内很强的共价键 使石墨具有很强的机械强度，可是石墨层间微弱的 分子键却使石墨层片极易完全解理，使石墨具有优 异的润滑性能。在使用过程中, 石墨即使被高速运 转的轮子划开, 仍然保持自己的共价键, 保持着良好 的机械强度，因此是耐腐蚀的性能优异的摩擦材料。

但是在实际应用时，石墨晶体的异向性经常会 带来一些危害, 因此, 需要采取一些方法减少石墨 材料的异向性。通过调节石墨晶体排布方向减少石 墨材料的性能异向性, 提高性能的均匀性, 主要通 过两种方法来实现：人为控制石墨鳞片的排布方向 或者将石墨片制备成球形石墨。

\subsection{1 通过调节石墨晶体排布方向改善石墨制品的} 均匀性

通过调节石墨晶体排布方向减少石墨材料的性 能异向性, 可以改善石墨性能的均匀性。石墨晶体 本身是片状结构, 受到外力作用, 必然会沿着与外 力垂直的方向定向排列。因此, 采用石墨特别是大 鳞片石墨作为原料制备石墨制品中, 石墨很容易定 向排列，导致石墨制品的性能呈现出异向性。

将制品放在液体中，采用等静压方式制备石墨 制品，可以避免石墨的异向性，即可获得各项同性
的石墨制品。通过调整石墨鳞片的方向性, 减少石 墨制品的异向性, 既可保证石墨优异性能的发挥, 又可避免石墨单晶的异向性。

采用普通制备方法获得的石墨制品中石墨晶体 的定向排列(图 6(a)), 而采用等静压方式制备的石 墨制品中石墨晶体不同方向排列的都有，虽然各个 石墨小晶体仍然呈各向异性，但是从宏观上看，形 成近似于乱层结构 “(tubostratic stacking)”石墨(图 6(b) $)^{[24]}$, 从而表现出良好的各向同性。

\subsection{2 将石墨鳞片制备成球形石墨减少石墨异向性}

石墨是柔性材料，很容易变形，采用球磨进行 球形化等粉体加工处理, 可以使鳞片状的石墨片转 化为球形石墨。球形化和分级处理后的石墨材料用 于锂电池的电极材料, 使锂离子电池性能得到很大 提高。

采用摚拌磨和微细粒子复合化, 对天然石墨进 行球形化整形，获得球化石墨，在锂离子电池等领 域得到广泛应用 ${ }^{[40-41]}$ 。但是, 工业球形石墨的制备 工艺复杂, 球形化产率较低, 球形石墨生产成本很 高，球形化过程中鳞片石墨浪费巨大。虽然现在通 过球磨工艺已经能够由石墨片生产出球形石墨, 但 是石墨球形化的理论依据还很不明确。石墨球形化 机理的深入研究有可能为球形石墨的研制、性能提 高提供理论依据。

\section{3 天然石墨的结构及组装状态}

\section{1 天然石墨的结构}

石墨具有多种同质多像体，从石墨晶体结晶学 角度来看, 至少存在两种晶体结构形式: (a)六方晶 系的六方石墨; (b)三方晶系的菱形石墨 ${ }^{[1,7,42]}$ (图 7)。

\section{2 天然石墨的自然组装}

事实上, 即使是常见的鳞片石墨, 其结晶颗粒、 结晶程度也会有所不同，鳞片的排布规律也会有很 大差异 ${ }^{[7]}$, 既可以是六次对称的六方晶体、三次对称
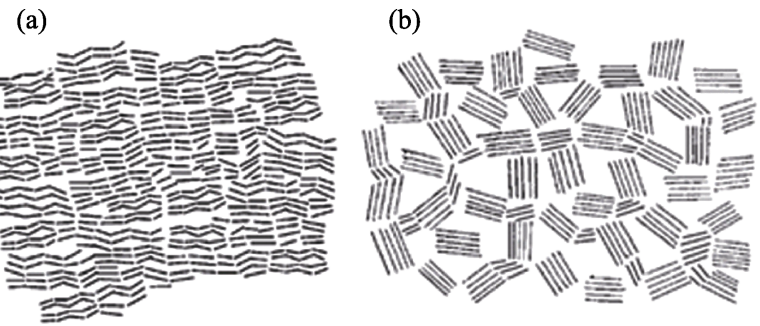

图 6 不同制备方法获得的石墨制品中的石墨的晶体排布特征 Fig. 6 Sketch of graphite crystalline arrangement in graphitic materials by different preparation

(a) Aanisotropic polycrystalline graphite; (b) Isotropic and tubostratic stacking polycrystalline graphite 


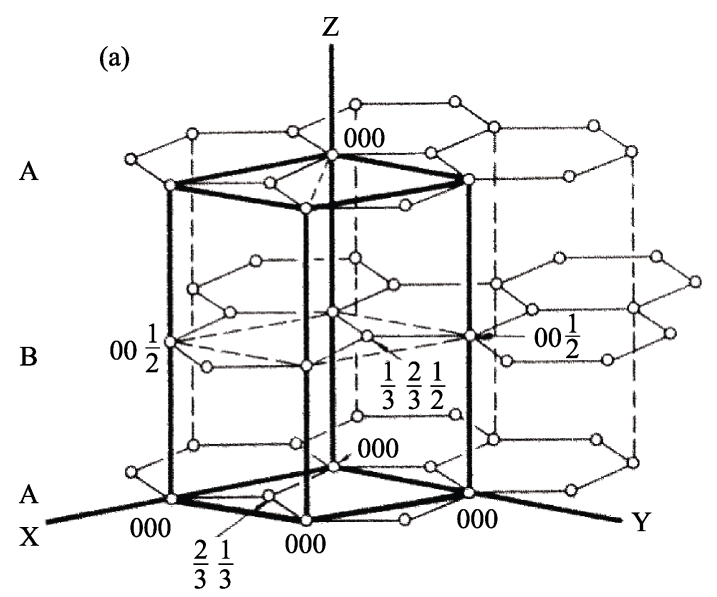

(b)

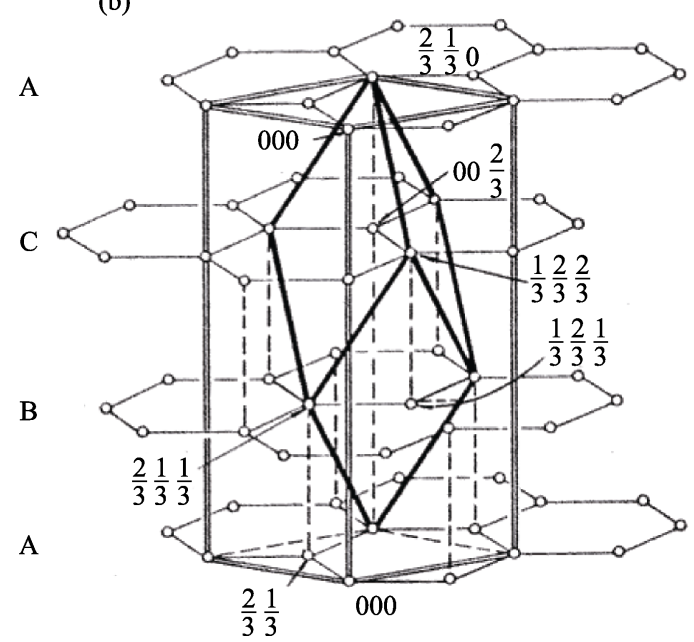

图 7 石墨的晶体结构

Fig. 7 Sketch of graphite crystal structure (a) Hexagonal; (b) Trigonal system

结构的菱面体晶体(图 7), 也可以形成完美的球形

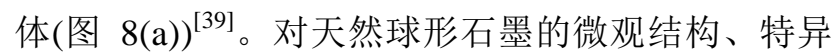
性能还有待进一步深入研究 ${ }^{[43]}$ 。球形石墨中存在雉 形石墨，通过电子显微技术可以发现在天然球形石 墨中也存在大量大小不等的球形化石墨颗粒, 天然 石墨中可能存在自然的微观组装结构(图 8(b))。对于 天然石墨微观组装结构和性能的深入研究, 可以为 工业球形石墨的制备工艺的改善、球形化率的提 高提供理论依据, 推动石墨矿物资源更有效的开发 利用。

\section{3 天然石墨的成因、结构和性能}

不同地方的石墨成矿机理存在差异, 有区域变 质型石墨矿、接触变质型隐晶质石墨矿和岩浆热液 型晶质石墨矿床三种成因。成因不同, 石墨的结构 性能存在差异, 其使用效能也必然有差异。工业上 石墨矿石仅仅分为晶质(鳞片状)石墨矿石和隐晶质 (土状)石墨矿石两种工业类型 ${ }^{[2,7]}$ 。

目前对于天然石墨的结构认识只有菱形和六方
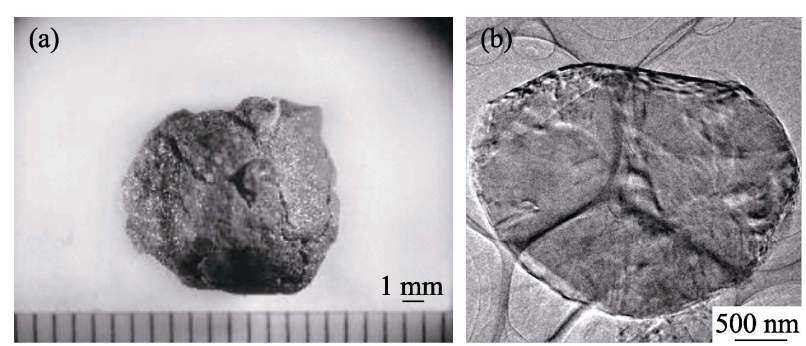

图 8 天然石墨自然组装形成的球形石墨 ${ }^{[39]}$

Fig. 8 Images of spherical graphite nanostructured naturally ${ }^{[39]}$ (a) Actual sample; (b) TEM image

两种石墨结构，事实上，自然界的石墨形成条件多 种多样, 科学研究已经证明也存在天然自组装的石 墨结构状态。

作为天然资源, 不同地区的石墨成因类型不同, 微观结构存在差异, 使其在晶体结构和晶体排布特 点上存在差异, 因此其性能, 特别是使用效能也必 然不同，进而决定其不同的工业价值和用途。在金 刚石合成方面, 与六方石墨相比, 菱形石墨更容易 制备金刚石、生产效率更高 ${ }^{[6-7]}$ 。

对于天然石墨的微观结构设计和物理化学性能 分析, 对于石墨的使用效能和实际应用有重要意 义。对于天然石墨的微结构和性能研究, 有可能开 拓天然石墨作为功能材料的巨大潜力。通过分析天 然石墨微观组装结构, 有可能推动石墨矿物资源的 有效开发利用。通过纳米结构组装、可以获得新型 材料 ${ }^{[1,5-6,44-45]}$, 通过石墨的微观结构设计, 可以调 整其性能, 设计新型石墨功能材料, 开发新型石墨 储能材料和石墨烯片材料。

\section{4 结束语}

本文在分析石墨微观结构、性能基础上, 提出 了石墨的纳米组装的概念, 认为可以采用多种方法 进行石墨的纳米结构组装: 采用制备石墨层间化合 物, 制备碳石墨合金等方法引入纳米功能粒子, 组 装碳石墨材料; 通过打开石墨层片制备纳米石墨烯 片; 通过制造孔隙结构增加活性空间; 通过制备球 形石墨，调节石墨晶体排布方向减少石墨材料的性 能异向性，提高性能的均匀性。目前对于天然石墨 的结构认识只有菱形和六方两种石墨结构, 可是自 然界的石墨形成条件多种多样, 还存在天然自组装 的石墨结构状态。通过分析天然石墨微观组装结构, 有可能推动石墨矿物资源更有效的开发利用。通过 石墨结构的纳米组装, 可以进行石墨的加工和改性, 获得更高性能的石墨材料制品, 开发石墨作为新型 碳功能材料的巨大潜力。 


\section{致谢}

杨扬同学帮助转换论文图形文件格式、并完善 论文参考文献格式，在此表示感谢！

\section{参考文献:}

[1] CHUNG D D L. Graphite. Journal of Materials Science, 2002, 37(8): 1475-1489.

[2] YI LI-WEN. Graphite resources and developments in the world. Land and Resources Information, 2011, 6: 29-32, 23.

[3] YI LI-WEN. Graphite, Land and Resources Information Center, Annual Review of World Mineral Resources. Beijing: Geological Publishing House, 2013: 286-292.

[4] CHUAN XIU-YUN. Graphite resources and its industries development analysis. High technology and Industry, 2014, 213: 50-55.

[5] CHUAN XIU-YUN, LU XIAN-CHU, GONG PING. Installing engineering in micro-struture and photocatalytic properties of porous mineral. Earth Science Frontiers, 2005, 12(1): 188-195.

[6] CHUAN XIU-YUN. Nanostructured engineering of mineral materials. Earth Science Frontiers, 2000, 7(Suppl): 58, 86.

[7] CHUAN XIU-YUN. Natural graphite mineral and energy storage materials. China Non-metallic Mining Industry Herald, 2013, 103(3): $1-3$.

[8] DRESSELHAUS M S, DRESSLHAUS G. Intercalation compounds of graphite. Advances in physics, 1981, 30(2): 139-142.

[9] FLANDROIS S, MASSON J M, ROUILLON J C, et al. Intercalation compounds of graphite with nickle chloride. Synthetic Metals, 1981, 3: 1-13.

[10] KANG FEI-YU. Research and application of graphite intercalation compounds. New Carbon Materials, 1998, 13(3): 65-66.

[11] CHUAN XIU-YUN, CHEN DAI-ZHAN, ZHOU XUN-RUO. Intercalation of $\mathrm{CuCl}_{2}$ into expanded graphite. Carbon, 1997, 353(2): 311-313.

[12] CHUAN XIU-YUN. The electrical properties of expanded graphite intercalation compounds. Journal of Materials Scicence \& Technology, 2001, 17(3): 371-375.

[13] CHUAN XIU-YUN. Structure, properties and application analysis of graphite intercalation compounds. New carbon materials, 1996, 11(2): $36-42$

[14] CHUAN XIU-YUN. Synthesis techniques and applications of graphite intercalation compounds. Nonmetallic Mines, 1997, 4: $18-25$.

[15] CHUAN XIU-YUN. Formation mechanism of graphite intercalation compounds. New Carbon Materials, 2000, 15(1): 50-56.

[16] CHUAN XIU-YUN. Micro-structures of the $\mathrm{CuCl}_{2}$-GICs on TEM. Journal of Inorganic Materials, 2000, 15(1): 79-87.

[17] CHUAN XIU-YUN. Magnetic properties of expanded graphite intercalation compound of $\mathrm{CuCl}_{2}-\mathrm{NiCl}_{2}$. Journal of Inorganic Materials, 2000, 15(6): 1077-1082.

[18] CHUAN XIU-YUN, CHEN DAI-ZHAN, ZHOU XUN-RUO. The electrical property and its mechanism of intercalation compounds of $\mathrm{CuCl}_{2}$. Acta Physica Sinica, 1999, 48(6): 1132-1136.

[19] CHUNG D D L. Exfoliation of graphite. Journal of Materials Science, 1987, 22(12): 4190-4198.

[20] YANG LI, LIU HONG-BO, LI FU-JIN, et al. Characterization and microwave absorption properties of ternary $\mathrm{FeCl}_{3}-\mathrm{NiCl}_{2}$-GICs. New Carbon Materials, 2008, 23(4): 367-373.

[21] ZHOU MING-SHAN, LI CHENG-JUN, XU MING, et al. Properties and preparation of graphite intercalation compound of $\mathrm{FeCl}_{3}-\mathrm{CrO}_{3}$-GIC. Chinese Journal of Inorganic Chemistry, 2006, 22(11): 2049-2054

[22] LIU HONG-BO, XIAO GU-YU, SU YU-CHANG, et al. Study on structural stability and thermostability of $\mathrm{FeCl}_{3}$-GIC. New Carbon Materials, 2000, 15(1): 18-22.
[23] REN HUI, JIAO QINGJIE, QIAO XIAOJING, et al. Synthesis and characterization of the ternary graphite intercalation compounds. Journal of Beijing Institute of Technology, 2003, 23(2): 248-251.

[24] MICHIO INAGAKI, KANG FEI-YU. Carbon Materials Science and Engineering from Fundamental to Application. Beijing: Tsinghua University Press, 2006: 29-31.

[25] LI SONG, ZHENG LIU, ARAVA LEELA MOHANA REDDY, et al. Binary and ternary atomic layers built from carbon, boron, and nitrogen. Advanced Materials, 2012, 24: 4878-4895.

[26] HAN PENG-YI. Microscopic fluctuations of graphene crystals. Physics, 2009, 38(6): 386.

[27] PARTOENS B, PETERS F M. From graphene to graphite: electronic structure around the K point. Phys. Rew. B, 2006, 74(7): 075404.

[28] GEIM A K, NOVOSELOV K S. The rise of graphene. Nature, 2007, 6: 183-191.

[29] CHUAN XIU-YUN. Graphene-like nanosheets synthesized by natural flaky graphite in Shandong, China. International Nano Letters, 2013, 3: 6-11.

[30] CHUAN XIU-YUN. Graphene like flakes by natural graphite flake in Shandong with microwave method. Acta Mineralogica Sinica, 2012, Sl: 87-88.

[31] ZHANG Q, ZHANG L, LI J H. Application of graphene gased nanomaterials in enzymatic electrochemical biosensor. Chinese Journal of Analytical Chemistry, 2013, 41(5): 641-649.

[32] SUN XIAO-DAN, LIU ZHONG-QUN, YAN HAO. Preparation and biological application of graphene quantum dots. Journal of Inorganic Materials, 2016, 31(4): 337-344.

[33] SUN GUILEI, YAN HONGHAO, LI XIAOJIE. Preparation of grapheme by detonation using liquid explosive. Explosion and Shock Waves, 2016, 36(5): 715-720.

[34] MALACHI NOKED, ABRAHAM SOFFER, DORON AURBACH. The electrochemistry of activated carbonaceous materials: past, present, and future. J. Solid State Electrochem., 2011, 15: 15631578.

[35] CHUAN XIU-YUN. Carbon materials applied in environmental engineering. Acta Petrologica et Mineralogica, 2001, 20(4): 507510.

[36] KANG FEI-YU. Graphite intercalation compound and exfoliated graphite. New Carbon Materials, 2000, 15(4): 80.

[37] KANG FEI-YU, ZHENG YONG-PING, ZHAO HENG, et al. Sorption of heavy oil and biomedical liquids into exfoliated graphite-research in China. New Carbon Materials, 2003, 18(3): 161173.

[38] CHUNG D D L. Carbon materials for structural self-sensing, electromagnetic shielding and thermal interfacing. Carbon, 2012, 50: 3342-3353.

[39] LIU HENG, SUN MINGQING, LI JUN, et al. Piezoresistive effects of cement-based composites containing graphene nanoplatelets. Journal of Functional Materials, 2015, 16: 16064-16068.

[40] YANG YU-FEN, CHEN XIANG-BIAO, GAI GUO-SHENG, et al. Preparation of spherical graphite. Journal of Process Engineering, 2004, 4(z1): 309-313.

[41] HAO XIANG-YANG, GAI GUO-SHENG, YANG YU-FEN, et al. Shape modification of natural graphite powders used in lithium ion batteries. Rare Metal Material and Engineering, 2005, 34(z1): 167-169.

[42] CHUAN XIU-YUN, WANG TONG-KUAN, JEAN-BAPTISTE DONNET. Stability and existence of carbyne with carbon chains. New Carbon Materials, 2005, 20(1): 83-92.

[43] CHUAN XIU-YUN, NOZOMI MORIHARA, BAO YING, et al. Orbicular graphite in Oshirabetsu, Hokkaido, Japan. Acta Geologica Sinica, 2012, 86(2): 241-246.

[44] CHUNG D D L. Self-Monitoring structural materials. Mater. Sci. Eng. Rev., 1998, R22(2): 57-78.

[45] XU LI, SHENG PENG, CHEN XIN, et al. Preparation and application of three-dimensional graphene nanospheres in lithium ion battery. Journal of Inorganic Materials, 2016, 31(9): 976-980. 